\title{
D- 二聚体水平对前列腺癌疾病进展评估的临床意义研究
}

\section{The Clinical Significance of D-dimer in the Evaluation of Prostate Cancer Progression}

\author{
陈继祥 \\ Jixiang Chen \\ 广西壮族自治区人民医院 中国·广西 南宁 530021 \\ The People's Hospital of Guangxi Zhuang Autonomous Region, Nanning, Guangxi, 530021, China
}

\begin{abstract}
摘 要: 目的: 通过检测前列腺癌患者术前常规凝血指标及 D- 二聚体水平, 探讨血将 D-二聚体水平对前列腺癌疾病进展评 估的临床意义。方法: 回顾性分析我院 2015 年 3 月至 2019 年 3 月病理确诊的 97 例前列腺癌患者和 127 例良性前列腺增生 患者术前常规凝血功能和血浆 D-二聚体水平, 研究血将 D-二聚体水平对前列腺癌疾病进展评估的临床意义。结果: 前列腺 癌组术前 D-二聚体水平 $(1.86 \pm 1.53 \mathrm{mg} / \mathrm{L})$, 明显高于良性前列腺增生组 $(0.38 \pm 0.18 \mathrm{mg} / \mathrm{L})(P<0.01)$; 前列腺癌组的亚分组中临 床分期为 III IV 期血浆 D- 二聚体水平 $(2.45 \pm 1.62 \mathrm{mg} / \mathrm{L})$ 较 I II 期 $(0.98 \pm 0.83 \mathrm{mg} / \mathrm{L})$ 高 $(P<0.01)$; 前列腺癌伴骨转移患者血浆 D- 二聚体水平 $(2.84 \pm 1.71 \mathrm{mg} / \mathrm{L})$ 高于不伴骨转移患者 $(1.39 \pm 1.20 \mathrm{mg} / \mathrm{L})(P<0.01)$; 以及前列腺癌 Gleason 评分高危组 $(\geqslant 8$ 分) 血 浆 D-二聚体水平 $(2.28 \pm 1.66 \mathrm{mg} / \mathrm{L})$ 高于中低危组 $(\leqslant 7$ 分) 血浆 D- 二聚体水平 $(1.42 \pm 1.43 \mathrm{mg} / \mathrm{L})(P<0.05)$, 提示血录 D-二聚体 水平随前列腺癌疾病进展而升高。结论: 前列腺癌患者术前血浆 D-二聚体水平较良性前列腺增生患者高, 且 D-二聚体水平 与临床病理分期、是否伴骨转移、Gleason 评分呈正相关。建议在临床诊疗过程中,将血浆 D-二聚体水平作为前列腺癌病情进 展评估的一项参考指标, 以期为前列腺癌患者拟定更准确、更合适的诊疗方案。
\end{abstract}

\begin{abstract}
Objective: To investigate the clinical significance of plasma D-dimer in the evaluation of prostate cancer progression by detecting preoperative routine coagulation indexes and D-dimer of the patients with prostate cancer. Methods: A retrospective analysis of 97 patients with prostate cancer and 127 patients with benign prostatic hyperplasia who were pathologically diagnosed in the people's hospital of Guangxi Zhuang autonomous region from March 2015 to March 2019 were retrospectively analyzed for preoperative routine coagulation function and plasma D-dimer, and to study the clinical significance of plasma D-dimer in evaluating the progression of prostate cancer. Results: The preoperative D-dimer $(1.86 \pm 1.53 \mathrm{mg} / \mathrm{L})$ of the prostate cancer group was significantly higher than that of the benign prostatic hyperplasia group $(0.38 \pm 0.18 \mathrm{mg} / \mathrm{L})(P<0.01)$. The plasma D-dimer $(2.45 \pm 1.62 \mathrm{mg} / \mathrm{L})$ of the clinical stage III $\sim \mathrm{IV}$ among the subgroups of the prostate cancer group is higher than that of stage I $\sim$ II $(0.98 \pm 0.83 \mathrm{mg} / \mathrm{L})(P<0.01)$. The plasma D-dimer in patients with prostate cancer with bone metastasis $(2.84 \pm 1.71 \mathrm{mg} / \mathrm{L})$ was higher than that in patients without bone metastasis $(1.39 \pm 1.20 \mathrm{mg} / \mathrm{L})(P<0.01)$, and the plasma D-dimer $(2.28 \pm 1.66 \mathrm{mg} / \mathrm{L})$ in the high-risk group of prostate cancer Gleason score ( $\geqslant 8$ points) was higher than that $(1.42 \pm 1.43 \mathrm{mg} / \mathrm{L})$ of the middle and low risk group ( $\leqslant 7$ points) $(P<0.05)$, suggesting that the plasma D-dimer increases with the progression of prostate cancer. Conclusion: The preoperative plasma D-dimer in patients with prostate cancer is higher than that in patients with benign prostatic hyperplasia, and the D-dimer is positively correlated with clinicopathological stage, bone metastasis and Gleason score. It is recommended that the plasma D-dimer be used as a reference index for the evaluation of the progress of prostate cancer in the clinical diagnosis and treatment process, in order to develop a more accurate and suitable diagnosis and treatment plan for the patients with prostate cancer .
\end{abstract}

关键词: D- 二聚体; 前列腺癌; 疾病进展

Keywords: D-dimer; prostate cancer; disease progression

DOI : $10.36012 /$ pmr.v2i3.2288

\section{1 引言}

恶性肿瘤患者往往伴随血液系统凝血功能和纤溶系统 功能障碍, 例如,动静脉血栓、迁移性血栓和弥漫性凝血功能
障碍 (DIC) 等血栓性疾病,均是癌症的重要并发症。肿瘤细胞 可直接或间接地影响凝血和纤溶功能而引起肿瘤细胞增殖、 局部浸润和转移叫。本研究通过回顾性分析前列腺癌患者术

【作者简介】陈继祥(1989 ), 男,广西贺州人, 从事前列腺癌相关渗疗研究。 
前常规凝血功能及血浆 D-二聚体水平, 研究探讨凝血功能 及纤溶功能变化在前列腺癌疾病进展评估的临床意义。

\section{2 资料与方法}

\section{1 一般资料}

选取广西壮族自治区人民医院 2015 年 3 月至 2019 年 3 月在本院泌尿外科住院诊治且病理确诊的 96 例前列腺癌患 者(观察组)和 126 例良性前列腺增生患者(对照组)。观察组 (均行超声引导下经直肠前列腺穿刺活检, 活检病理确诊为 前列腺癌)平均年龄 $(69.63 \pm 6.58)$ 。观察组中按 2002 年 AJCC 分期系统: III IV 期 57 例, 平均年龄 $(70.18 \pm 6.18)$; I II 期 39 例, 平均年龄 $(68.82 \pm 7.13)$; 伴有骨转移患者 31 例, 平均 年龄 $(70.55 \pm 6.53)$, 不伴骨转移患者 65 例, 平均年龄 $(69.18 \pm$ 6.61 ); Gleason 评分高危组 ( $\geqslant 8$ 分 ) 66 例, 平均年龄 $(70.29 \pm$ $6.47)$, 中低危组 30 例 ( $\leqslant 7$ 分), 平均年龄 $(68.17 \pm 6.71)$ 。对 照组 126 例均为良性前列腺增生患者 (在本院住院行经尿道 前列腺电切术后病理证实), 平均年龄 $(69.71 \pm 7.70)$ 。血样为 入院次日清晨空腹采集。入组标准: 观察组均行超声引导下 经直肠前列腺穿刺活检, 活检病理确认为前列腺癌; 对照组 均行经尿道前列腺电切术, 术后病理证实为良性前列腺增生 $(\mathrm{BPH})$ 。排除标准: (1)患者既往伴随有血液及其他凝血功能 障碍疾病; (2)患者伴随有心脏瓣膜性疾病、心律失常、脑梗 塞、糖尿病等可能影响凝血功能的疾病; (3)患者一个月内有 口服抗凝药物; (4)患者患有其他恶性肿瘤或者有放化疗病 史; (5)患者有长期吸烟史。6 患者一周内有发热、感染等。(7)患
者合并有其他严重心肺肝肾功能损害等。

\section{2 方法}

所有患者血样采集为入院次日空腹抽取静脉血 $3 \mathrm{ml}$, 并 联合使用 $0.1 \mathrm{~mol} / \mathrm{L}$ 枸椽酸钠抗凝管, 采用 $3000 \mathrm{r} / \mathrm{min}$ 全自动 血凝分析仪离心 $10 \mathrm{~min}$ 。

\section{3 观察指标}

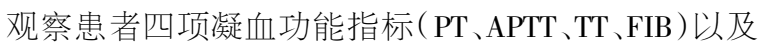
D-D 水平。

\section{4 统计学处理}

采用 SPSS 17.0 版分析软件。各组计量资料用均值土标准 差 $(\bar{x} \pm s)$ 表示, 组间比较用独立样本 $t$ 检验。以 $P \leqslant 0.05$ 为差 异有统计学意义。

\section{3 结果}

前列腺癌患者与良性前列腺增生患者凝血功能指标对 比, 两组 PT、APTT、TT、FIB 水平无显著性差异 $(P>0.05)$; D二聚体水平差异有统计学意义 $(P<0.01)$ ( 见表 1$)$ 。

前列腺癌组亚分组中分期为 III $\sim$ IV 期与 I II 期患者凝 血功能指标对比( 见表 2)。PT、APTT、TT、FIB 水平无显著性 差异 $(P>0.05), \mathrm{D}-$ 二聚体水平差异有统计学意义 $(P<0.01)$ 。

前列腺癌组中伴有骨转移与不伴骨转移患者凝血功能 指标对比(见表 3 )。PT、APTT、TT、FIB 水平无显著性差异 $(P>$ $0.05), \mathrm{D}-$ 二聚体水平差异有统计学意义 $(P<0.01)$ 。

前列腺癌组中 Gleason 评分高危组 ( $\geqslant 8$ 分) 与中低危组

表 1 前列腺癌患者与前列腺增生患者凝血功能指标对比 $(\bar{x} \pm s)$

\begin{tabular}{c|c|c|c|c|c|c|c}
\hline 组别 & $\mathrm{N}$ & 年龄 & $\mathrm{PT}(\mathrm{s})$ & $\mathrm{APPT}(\mathrm{s})$ & $\mathrm{TT}(\mathrm{s})$ & $\mathrm{Fib}(\mathrm{g} / \mathrm{L})$ & $\mathrm{D}-$ 二聚体 $(\mathrm{g} / \mathrm{L})$ \\
\hline 前列腺癌组 & 96 & $69.63 \pm 6.58$ & $13.44 \pm 1.63$ & $36.83 \pm 3.68$ & $16.77 \pm 1.34$ & $3.96 \pm 0.98$ & $1.86 \pm 1.53$ \\
\hline 前列腺增生组 & 126 & $69.71 \pm 7.70$ & $13.30 \pm 0.57$ & $37.15 \pm 3.60$ & $16.66 \pm 1.02$ & $3.80 \pm 0.97$ & $0.38 \pm 0.18$ \\
\hline$P$ & & $(\mathrm{P}>0.05)$ & $(\mathrm{P}>0.05)$ & $(\mathrm{P}>0.05)$ & $(\mathrm{P}>0.05)$ & $(\mathrm{P}>0.05)$ & $(\mathrm{P}>0.01)$ \\
\hline
\end{tabular}

表 2 前列腺癌组中分期为 III IV 期患者与 I II 期患者凝血能指标对比 $(\bar{x} \pm s)$

\begin{tabular}{c|c|c|c|c|c|c|c}
\hline 组别 & $\mathrm{N}$ & 年龄 & $\mathrm{PT}(\mathrm{s})$ & APPT $(\mathrm{s})$ & $\mathrm{TT}(\mathrm{s})$ & $\mathrm{Fib}(\mathrm{g} / \mathrm{L})$ & $\mathrm{D}-二$ 聚体 $(\mathrm{g} / \mathrm{L})$ \\
\hline $\mathrm{III} \sim \mathrm{IV}$ 期患者 & 57 & $70.18 \pm 6.18$ & $13.69 \pm 1.25$ & $37.08 \pm 3.98$ & $16.58 \pm 1.0$ & $3.89 \pm 0.93$ & $2.45 \pm 1.62$ \\
\hline $\mathrm{I} \sim \mathrm{II}$ 期患者 & 39 & $68.82 \pm 7.13$ & $13.07 \pm 2.02$ & $36.46 \pm 3.19$ & $17.04 \pm 1.70$ & $4.05 \pm 1.05$ & $0.98 \pm 0.83$ \\
\hline$P$ & & $(\mathrm{P}>0.05)$ & $(\mathrm{P}>0.05)$ & $(\mathrm{P}>0.05)$ & $(\mathrm{P}>0.05)$ & $(\mathrm{P}>0.05)$ & $(\mathrm{P}>0.01)$ \\
\hline
\end{tabular}

表 3 前列腺癌组中伴有骨转移与不伴骨转移患者凝血功能指标对比 $(\bar{x} \pm s)$

\begin{tabular}{c|c|c|c|c|c|c|c}
\hline 组别 & $\mathrm{N}$ & 年龄 & $\mathrm{PT}(\mathrm{s})$ & $\mathrm{APPT}(\mathrm{s})$ & $\mathrm{TT}(\mathrm{s})$ & $\mathrm{Fib}(\mathrm{g} / \mathrm{L})$ & $\mathrm{D}-$ 二聚体 $(\mathrm{g} / \mathrm{L})$ \\
\hline 伴骨转移患者 & 31 & $70.55 \pm 6.53$ & $13.79 \pm 1.27$ & $36.95 \pm 3.63$ & $16.79 \pm 1.17$ & $4.11 \pm 0.95$ & $2.84 \pm 1.71$ \\
\hline 不伴骨转移患者 & 65 & $69.18 \pm 6.61$ & $13.27 \pm 1.76$ & $36.78 \pm 3.72$ & $16.76 \pm 1.43$ & $3.88 \pm 0.99$ & $1.39 \pm 1.20$ \\
\hline$P$ & & $(\mathrm{P}>0.05)$ & $(\mathrm{P}>0.05)$ & $(\mathrm{P}>0.05)$ & $(\mathrm{P}>0.05)$ & $(\mathrm{P}>0.05)$ & $(\mathrm{P}>0.01)$ \\
\hline
\end{tabular}


表 4 前列腺癌组 GI eason 评分高危组 ( $\geqslant 8$ 分) 与中低危组 ( $\leqslant 7$ 分) 患者凝血功能指标对比 $(\bar{x} \pm s$ )

\begin{tabular}{c|c|c|c|c|c|c|c}
\hline 组别 & $\mathrm{N}$ & 年龄 & $\mathrm{PT}(\mathrm{s})$ & $\mathrm{APPT}(\mathrm{s})$ & $\mathrm{TT}(\mathrm{s})$ & $\mathrm{Fib}(\mathrm{g} / \mathrm{L})$ & $\mathrm{D}-$ 二聚体 $(\mathrm{g} / \mathrm{L})$ \\
\hline 高危组患者 $(\geqslant 8$ 分 $)$ & 66 & $70.29 \pm 6.47$ & $13.48 \pm 1.91$ & $36.99 \pm 3.91$ & $16.72 \pm 1.24$ & $3.99 \pm 0.93$ & $2.28 \pm 1.66$ \\
\hline 中低危组患者 $(\leqslant 7$ 分 $)$ & 30 & $68.17 \pm 6.71$ & $13.34 \pm 0.71$ & $36.49 \pm 3.14$ & $16.87 \pm 1.5$ & $3.89 \pm 1.09$ & $1.42 \pm 1.43$ \\
\hline$P$ & & $(\mathrm{P}>0.05)$ & $(\mathrm{P}>0.05)$ & $(\mathrm{P}>0.05)$ & $(\mathrm{P}>0.05)$ & $(\mathrm{P}>0.05)$ & $(\mathrm{P}>0.05)$ \\
\hline
\end{tabular}

（\$7 分）对比（表 4）PT、APTT、TT、FIB 水平无显著性差异 $(\mathrm{P}>0.05), \mathrm{D}-$ 二聚体水平差异有统计学意义 $(P<0.05)$ 。

\section{4 讨论}

近年来, 曾有相关研究显示, 各类肿瘤患者都伴有凝血 系统激活。曾有文献报道, 50\%的恶性肿瘤患者和 $90 \%$ 癌症 伴有转移的患者出现一个或多个凝血参数异常 ${ }^{[2,3]}$ 。恶性循环 性肿瘤具有多种生物学特性, 有研究认为可能癌组织本身含 有促凝活性及纤溶性成分所致血凝和纤溶的异常, 从而导致 血浆 D-二聚体水平明显升高 ${ }^{[4]}$ 。随着疾病进展, 恶性肿瘤患 者血浆 D-二聚体水平升高, 反映机体内凝血和纤溶系统的 活性增强, 机体内高凝状态加重, 易发生血栓栓塞和导致弥 散性血管内凝血 ${ }^{[5]}$ 。

血浆 D-二聚体是一项特异性的纤溶过程标记物, 首先 由纤维蛋白单体与活化因子 X III交联,再被纤溶酶降解所产 生的一种特异性产物, 其血浆水平升高是继发性纤溶激活的 特征反映 ${ }^{[6]}$ 。有研究认为, 肿瘤细胞不仅能激活凝血系统, 还 可以损伤血管内皮细胞, 从而引起血小板活性增强和纤溶蛋 白溶解异常 ${ }^{[7,8]}$ 。Khoury 等 ${ }^{[9]}$ 对 28 例难治性前列腺癌进行血 浆 D-二聚体定量测定, 发现 D-二聚体水平都升高了; 还有 研究报道, 在与前列腺癌的相关性方面, D-二聚体水平和 tPSA 的检测较肿瘤细胞计数更具有优势, 建议 D-二聚体水 平升高可作为前列腺癌疾病进展的重要指标 ${ }^{[10,11]}$ 。

本研究中,由表 1 可知, 前腺癌患者血浆 D-二聚体水平 明显高于前列腺增生患者 $(P<0.01)$, 这一结果与 Khoury 等 研究结果相符, 本研究还将观察组 (前列腺癌组) 与对照组 (前列腺增生组) 血浆中 PT、APTT、TT、FIB 水平相比较, 发现 统计结果无显著性差异 $(P>0.05)$ 。表明前列腺癌患者存在凝 血及纤溶系统激活。

另外, 在观察组的亚分组中, 由表 2 结果显示, 分期为 III $\sim \mathrm{IV}$ 期患者血浆 D-二聚体水平比 I II 期患者高 $(P<0.01)$, 两组 PT、APTT、TT、FIB 水平相比较, 统计学无显著性差异 $(P>0.05)$; 表 3 研究资料中还将Gleason 评分为高危组 $(\geqslant 8$
分）的患者血浆 D-二聚体水平和 Gleason 评分为中低危组 ( $\leqslant 7$ 分) 患者血浆 D-二聚体水平相比较, 发现高危组患者 血浆 D-二聚体水平高于中低危组, 统计学有显著性差异 $(P<$ 0.05 ), 两组 PT、APTT、TT、FIB 水平相比较, 统计学无显著性 差异 $(P>0.05)$; 表 4 中研究结果显示前列腺癌伴骨转移患者 血浆 D-二聚体水平较不伴骨转移患者高, 统计学有显著性 差异 $(P<0.05)$, 两组 PT、APTT、TT、FIB 水平相比较, 统计学 无显著性差异 $(P>0.05)$ 。本研究结果与王素侠 ${ }^{[12]}$ 等人研究结 果相符合, 表明前列腺癌患者随着病情进展, 血浆 D-二聚体 水平升高呈正相关, 提示前列腺癌患者纤溶功能障碍的情况 愈发严重。

目前, 临床上对前列腺癌进展、具体病灶控制及预后评 估诊断尚缺乏统一的检测标准, 如 PSA 只是评价前列腺癌 的诊断和治疗后疾病的进展指标之一, 无法准确评估前列腺 癌术前或者治疗前疾病进展情况 ${ }^{[13]}$ 。本研究结果显示前列腺 癌患者 D-二聚体水平较前列腺增生患者高, 并且前列腺癌 患者血浆 D-二聚体水平与前列腺癌疾病进展呈正相关。

综上所述, 本研究结果提示, D-二聚体水平与前列腺癌 病情进展呈正相关, 预示着在临床诊疗过程中, 是否可以将 前列腺癌患者术前血浆 D-二聚体水平作为前列腺癌患者病 情进展的一项判断指标, 这也许能为前列腺癌患者病情进展 评估、围手术期安全评估、治疗指导等提供更加有力的支持。 但是术前血浆 D-二聚体水平与前列腺癌患者总生存时、术 后生存时间、内分泌治疗后生存时间等相关问题仍需进一步 研究。

\section{参考文献}

[1] Glassman AB,Jones E.Thrombosis and coagulation abnomalitises associated with cancer [J].Ann Clin Lab Sci,1994 (24):1-5 .

[2] Rickles FR,Levine MN.Epidemicology of thrombosis in cancer[J].Acta Haematol,2002(106):6-12.

[3] Rickles FR,Levine MN,Dvorak HF.Abnamalities of hemastasis in malignancy. In:Colman RW,Hirsh J, 
Marder VJ,eds.Hemostasis and Thrombosis.Basic principles \&clinical practice [J].4th ed.Philadelphia: Lippincott Williams \&Wilkins,2001(1):1131-1152.

[4] Olsson E,Svartling N,Asko-Seljavaara S,etal.Activation of coagulation and fibrinolysis during reconstructive microsurgery in patients with cancer [J].Microsurgery, 2001(21):208-213.

[5] Kono T,Ohtsuki T,Hosomi N,etal.Clinical characteris tics of ischemic stroke in elderly patients with cancer[J]. Nihon Ronen Igakkai Zasshi,2011,48(1):57-62.

[6] Cushman M,Folsom R,Wang L,etal.Fibrin fragment D dimer and the risk of future venous thrombosis[J].Blood, 2003,101(4):1243-1248.

[7] Riedl J,Pabinger I,Ay C.Platelets in cancer and thrombosis[J]. Hamostaseologie, 2014,34(1):54-62.

[8] John A. Cancer and venous thromboembolism :scope of the problem[J]Cancer Control,2005,12(1):5-10.

[9] Khoury JD,Adcock DM,Chan F, etal.Increases in quantitative D dimer levels correlate with progressive disease better than circulating tumor cell counts in patients with refractory prostate cancer $[\mathrm{J}]$.Am J Clin Pathol,2010,134(6):964-969.

[10] Deme D,Ragán M,Kalmár K,etal.Metastatic prostate cancer complicated with chronic disseminated intravascular coagulopathy causing acute renal failure, mimicking thrombotic thrombocytopenic purpura and hemolytic uremic syndrome:pathomechanism, differential diagnosis and therapy related to a case [J].Magyar Onkologia,2010,54(4):351-357.

[11] Bergamini S,Bellei E, Bonetti LR,et al.Inflammation: animportant parameter in the search of prostate cancer biomarkers[J]. Proteome Sci,2014,12(1):1-12.

[12] 王素侠,范志伟.前列腺癌疾病进展中 D.二聚体检测的意义 $[\mathrm{J}]$. 检验医学与临床,2016,13(24):3541-3543.

[13] 陈涛,邱建宏, 刘健.血清 GPc-3 联合 PSA 检测在前列腺癌诊 断中的临床意义[J].临床误诊误治,2016,29(9):105-107.

\section{（上接第 39 页）}

VSD 的认识不足, 因此护理人员在此过程中尤为重要。作为 第一线的工作人员，护理人员与患者面对面的时间较长, 对 患者的观察较医生有着优势, 对于患者的宣教与交流, 在术 后的及时发现与反馈, 对于临床上患者的治疗及康复不可或 缺 ${ }^{[4]}$ 。本次实验也存在着许多不足, 如由于患者在教育水平上 的差异, 有些患者在护士进行积极宣教后仍对宣教内容存在 不解, 以致出现 VSD 装置故障, 影响实验的客观性与准确 性, 因此面对不同教育水平的患者, 护理人员应采用因人而 异的沟通方式，以确保临床工作落到实处而不是敷衍了事。 可喜的是,近些年随着医疗水平的不断提高, 护理人员的医 疗技能越来越全面, 与医师的临床工作交集逐渐增多, 其与 医师工作的配合越来越默契, 减轻了医师的压力, 同时有利 于患者的康复, 这无疑在促进医疗发展及加强医护合作的道
路上更进了一步, 相信在不久的将来, 护理工作会扮演更为 重要的角色,在病患的诊治及康复过程中为患者提供更好的 服务 ${ }^{[4]}$ 。

\section{参考文献}

[1] 韦艳艳,罗毅,龙春萍,等.自创简易 VSD 联合早期程序化综合治 疗在中华眼镜蛇咬伤中的应用 [J].当代护士(中旬刊),2020,27 (3):71-73.

[2] 刘慧,冀永峰.VSD 治疗创面的护理研究[J].心理月刊,2020,15 (1): 126 .

[3] 朱天存.游离植皮术后 VSD 的应用及护理对策分析[J].名医, 2020(1):224.

[4] 杨琴,陈扶荣.浅论 VSD 治疗技术的术前术后护理要点[J].新疆 中医药,2019,37(6):74-75. 